\title{
Serotonin transporter (5-HTTLPR) genotype and childhood trauma are associated with individual differences in decision making
}

\section{Scott F. Stoltenberg ${ }^{*}$, Melissa K. Lehmann ${ }^{2}$, Cynthia Anderson ${ }^{2}$, Parthasarathi Nag $^{3}$ and Cheryl Anagnopoulos ${ }^{4}$}

${ }^{1}$ Behavior Genetics Laboratory, Department of Psychology, University of Nebraska-Lincoln, Lincoln, NE, USA

${ }^{2}$ Center for Conservation of Biological Resources, Department of Biology, Black Hills State University, Spearfish, SD, USA

${ }^{3}$ Department of Mathematics, Black Hills State University, Spearfish, SD, USA

${ }^{4}$ Department of Psychology, Black Hills State University, Spearfish, SD, USA

Edited by:

Jeanne M. McCaffery, Brown Medical

School, USA

Reviewed by:

Robert Philibert, The University of

Iowa, USA

David Meyre, McMaster University,

Canada

*Correspondence:

Scott F. Stoltenberg, Behavior

Genetics Laboratory, Department of

Psychology, University of

Nebraska-Lincoln, 238 Burnett Hall,

Lincoln, NE 68588-0308, USA.

e-mail: sstoltenberg2@unl.edu
The factors that influence individual differences in decision making are not yet fully characterized, but convergent evidence is accumulating that implicates serotonin (5-HT) system function. Therefore, both genes and environments that influence serotonin function are good candidates for association with risky decision making. In the present study we examined associations between common polymorphisms in the serotonin transporter gene (SLC6A4; 5-HTTLPR and rs25531), the experience of childhood trauma and decision making on the lowa gambling task (IGT) in 391 (64.5\% female) healthy Caucasian adults. Homozygosity for the 5-HTTLPR L allele was associated with riskier decision making in the first block of 20 trials (i.e., decision making under ambiguity, $p=0.004$ ). In addition, mean IGT performance was significantly worse in blocks 3-5 (i.e., decision making under risk, $p \leq 0.05$ ) for those participants who reported experiencing higher levels of childhood trauma. Our findings add to the growing evidence that genetic variation in the 5-HT system is associated with individual differences in decision making under ambiguity; and we report that the experience of childhood trauma is associated with relatively poor decision making under risk.

Keywords: SERT, rs25531, early life stress, ambiguity, risk

\section{INTRODUCTION}

There are individual differences in the capacity to make good decisions. Recently, efforts to identify factors that contribute to those individual differences in decision making have been increasing (Rogers, 2011). Such work is interdisciplinary and crosses levels of analysis from genotype to brain function to behavior (Schonberg et al., 2011). In addition to the recent emphasis on biological correlates of decision making, research on potentially influential psychosocial factors has been on the rise (Overman et al., 2010). It is likely that a better understanding the biopsychosocial architecture of decision making will have a broad impact on both theory and applications development in areas such as psychology, psychiatry, neuroscience, economics, and law (Glimcher et al., 2008).

The first wave of genetic analyses to focus on decision making has largely, but not solely, focused on candidate genes in neurotransmitter systems and on a now widely used behavioral task called the Iowa gambling task (Bechara et al., 1997), hereafter referred to as the IGT. The IGT was developed as a tool to examine decision making in clinical populations and is available as a component of a clinical assessment battery (Bechara, 2007), although it is increasingly being used to study individual differences in decision making in non-clinical (i.e., healthy) populations.
The IGT is a deceptively complex task that involves learning which decks produce desirable outcomes across 100 trials. Participants are instructed that the purpose of the task is to try to win money and that some decks of cards (depicted on a computer monitor) are "good" and others are "bad." The participant is left to decide what constitutes a "good" or a "bad" deck, but the assumption is that a good deck is one that helps to achieve the goal of winning money. So, in the IGT, the participant must first learn by experience which decks are advantageous before they can then go on to try to maximize their winnings. Therefore, performance on the IGT comprises two phases (Bechara et al., 1997; Brand et al., 2006, 2007a). The first is referred to as decision making under ambiguity, because in these early trials, the participant does not have direct knowledge of which decks are advantageous and which are disadvantageous. An interesting aspect of the task is that the "bad" decks are initially "good" in that they result in relatively large monetary gains; for example, the first eight cards from the $\mathrm{B}^{\prime}$ deck produce only gains. The second phase of the IGT consists of those trials for which the participant has some understanding of which decks are good and which are bad. There is some controversy regarding how much participants know about the response contingencies of each deck and when they know it (Brand et al., 2006). Although consensus has not yet been reached on how to define these two phases of decision making, emerging evidence 
suggests that at least in healthy adults only the first 20 trials should be considered ambiguity (Gendle and Golding, 2010; Stoltenberg and Vandever, 2010).

The serotonin (5-HT) neurotransmitter system has recently received considerable attention regarding its role in individual differences in decision making and it appears that 5-HT may be involved in learning from punishment (Rogers, 2011). It seems reasonable then that the influence of the 5-HT system on IGT performance would be seen during the early phase of the task when participants are learning which decks are advantageous and which are not. Altering the availability of serotonin by either acute depletion of its amino acid precursor tryptophan or by administration of the 5-HT precursor 5-Hydroxytryptamine can indicate the involvement of the 5-HT system in a given behavior. Acute tryptophan depletion reduces sensitivity to punishment-based information in decision making (Blair et al., 2008), and enhancing 5-HT availability impairs decision making under ambiguity, but not under risk (Gendle and Golding, 2010). Such findings implicate the 5-HT system in specific aspects of decision making and suggest that genes harboring polymorphisms that impact 5-HT availability are good candidates for further study.

Several recent candidate gene association studies have sought to determine whether specific genetic polymorphisms are associated with individual differences in aspects of decision making. Polymorphisms in tryptophan hydroxylase genes (the rate limiting enzyme in 5-HT synthesis) are associated with probabilistic choice in a gambling task (TPH2; Juhasz et al., 2010) and with decision making in women with bipolar disorder (TPH1; Maurex et al., 2009). Most of the focus in terms of serotonin system candidate genes, however, has been on the well-studied 5-HTTLPR promoter region polymorphism. Significant genotype effects on IGT performance have been reported for: patients with unipolar major depression $(N=124)$ in Hungary (Must et al., 2007); patients with obsessive compulsive disorder $(N=49)$ in Brazil (Da Rocha et al., 2008); non-depressed suicide attempters $(N=163)$ in France; healthy women $(N=88)$ in the Netherlands (Homberg et al., 2008); and healthy undergraduate students in Korea $(N=173$; Ha et al., 2009) China ( $N=572$; He et al., 2010) and the United States of America ( $N=188$; Stoltenberg and Vandever, 2010). Differences in the populations under study, the details of the specific IGT version and the definition of decision making under ambiguity and risk make it difficult to draw firm conclusions regarding the association between 5-HTTLPR genotype and decision making. However, there is converging evidence that 5-HTTLPR genotype is associated with IGT performance, but that the effect might be moderated by ethnicity, gender, mental health status and phase of decision making.

The seminal work of Caspi et al. (2003) has focused attention to the potential for 5-HTTLPR genotypes to be differentially sensitive to early life stressors. There appears to be convergent evidence that the S allele of 5-HTTLPR, when coupled with early life stress, results in enhanced vulnerability to problems later in life (Caspi et al., 2010). We have been unable to find studies that have investigated the potential influence of this gene $x$ environment interaction on decision making in healthy adults. Early life stress affects hippocampal function and cognition (Ivy et al., 2010), cognitive control (Mueller et al., 2010), and stress reactivity (Heim and Nemeroff, 2002); and is a risk factor for sexual risk taking for women (Hillis et al., 2001); depression, addiction, and other adverse outcomes (Anda et al., 2006). It seems reasonable to hypothesize that early life stressors could affect decision making as assessed by the IGT, but we were unable to find other studies that have directly addressed this question.

In this study, our aim is to replicate our earlier finding of an effect of 5-HTTLPR on decision making under ambiguity (i.e., the first 20 IGT trials; Stoltenberg and Vandever, 2010) in a larger independent sample. We report extending those findings to include the SLC6A4 (serotonin transporter) polymorphisms and rs25531 to better characterize the genetic variation in SERT. We also extend the findings to determine whether exposure to childhood trauma differentially influences decision making. We examine overall choice patterns using the probability of choosing from a good deck across trials to empirically determine the border between ambiguity and risk. We also examine the potential influence of gambling attitudes and beliefs and gambling problems on decision making. And finally, we also aim to replicate and extend our understanding of the influence of impulsivity on IGT performance.

\section{MATERIALS AND METHODS PARTICIPANTS AND BASIC PROCEDURE}

College students from a small Midwestern university $(N=477)$ were recruited via posters and brief in-class presentations that described the study. The local institutional review board approved the study and all participants gave informed consent prior to their participation. Participants completed questionnaires assessing impulsivity and health-risk behaviors, computer tasks assessing impulsivity and decision making and donated buccal cell samples for genotyping. Participants were remunerated \$20. Only data directly relevant to the questions under study are reported here; other analyses will be reported elsewhere. Data from 38 participants were excluded from analyses to reduce the risk of population stratification (i.e., we excluded those who did not indicate "White" race/ethnicity). We also exclude from these analyses those who had missing data for variables of interest (48). Only data from those will complete data $(N=391)$ is reported.

\section{BEHAVIORAL TASKS}

All computer tasks were administered on a standard desktop computer with a cathode ray tube display with a refresh rate of $60 \mathrm{~Hz}$. Participants completed the $\mathrm{A}^{\prime} \mathrm{B}^{\prime} \mathrm{C}^{\prime} \mathrm{D}^{\prime}$ version of the IGT (supplied by Dr. Bechara), with standard instructions read aloud by a research assistant (Bechara et al., 1997, 2000). No monetary reward was provided for performance. In the $\mathrm{A}^{\prime} \mathrm{B}^{\prime} \mathrm{C}^{\prime} \mathrm{D}^{\prime}$ version, decks $\mathrm{A}^{\prime}$ and $\mathrm{B}^{\prime}$ are considered disadvantageous and decks $\mathrm{C}^{\prime}$ and $\mathrm{D}^{\prime}$ are considered advantageous. Sixty cards were available in each computerized deck. Deck A' is characterized by relatively large average gains ( $\$ 125)$ and frequent (75\% of cards) but relatively moderate (\$250) average losses. Deck B' is characterized by relatively large average gains ( $\$ 125)$ and infrequent (10\% of cards) but relatively large ( $\$ 1875)$ average losses. Deck $C^{\prime}$ is characterized by relatively small average gains (\$62.50) and frequent (75\% of cards) but relatively small ( $\$ 41.67)$ average losses. Deck $D^{\prime}$ is characterized by 
relatively small average gains $(\$ 62.50)$ and infrequent $(10 \%$ of cards) but relatively moderate $(\$ 312.50)$ average losses.

The Stop Task was administered using ePrime (Psychology Software Tools, Sharpsburg, PA, USA; Logan et al., 1997). Participants were first given 32 training trials for the simple reaction time (RT) task of pressing the "enter" key when an X was presented in the center of the screen or pressing the " $m$ " key when an $\mathrm{O}$ was presented. The instructions given were to respond as rapidly and as accurately as possible. Stimuli were presented for $1,000 \mathrm{~ms}$ following a fixation point that was presented for $500 \mathrm{~ms}$. The participants were then instructed that on some of the trials they would hear a brief tone $(1,000 \mathrm{~Hz})$ and on those trials, they were to not respond to the visual stimulus. The participants were given 32 training trials of the full task (i.e., the simple RT task with the stop signal on $25 \%$ of trials). The stop signal delay was initially set at $250 \mathrm{~ms}$ following the stimulus presentation (i.e., $\mathrm{X}$ or $\mathrm{O}$ ) and adjusted dynamically after each stop trial depending on whether the participant inhibited successfully so that overall the participant should be successful on $50 \%$ of the stop trials. The next trial after a successful inhibition the stop signal delay was increased by $50 \mathrm{~ms}$ (making it more difficult to inhibit) and after a failure to inhibit the stop signal delay was decreased by $50 \mathrm{~ms}$. Participants then completed eight blocks of 32 trials for a total of 256 trials (192 go trials and 64 stop trials) with brief breaks between each block. Equal numbers of $\mathrm{X}$ and $\mathrm{O}$ stimuli were presented in random order. Stop signal reaction time (SSRT) was calculated by subtracting the average stop signal delay from the mean RT on go trials (Logan et al., 1997).

Participants also completed the Time Paradigm (Dougherty et al., 2005), which is a behavioral assessment of the passage of time. Participants were asked to estimate the passage of $60 \mathrm{~s}$ five times. Participants were instructed to press the space bar to start the timer and to press it again when they thought that $60 \mathrm{~s}$ had passed. We analyzed the mean of the five estimates. Participants did not receive any feedback regarding the accuracy of their estimates or any payments based on their performance.

\section{SELF-REPORT MEASURES}

The Gambling Attitudes and Beliefs Scale is a 35-item self-report questionnaire that assesses personal attitudes toward gambling (Strong et al., 2004). Each question is scored on a four-point scale ranging from strongly agrees to strongly disagree. The GABS include questions that involve cognitive biases, irrational beliefs about gambling, positively valued attitudes to gambling, and degree of subjective arousal, or excitement involving gambling. Although the GABS has no set scoring, the higher the score denotes that gambling is exciting and socially meaningful to the person and that luck and strategies are important. Validity of the GABS was tested by correlating the GABS scores to the South Oaks Gambling Screen (SOGS). The tests were correlated at $(r=0.40, p<0.001$; Strong et al., 2004).

The SOGS is a 26-item self-report questionnaire that assesses pathological gambling (Lesieur and Blume, 1987). The SOGS does not correspond exactly with the DSM-IV criteria for pathological gambling because it focuses on the maladaptive social and financial behavior of pathological gambling rather than the emotional components. The SOGS does, however, differentiate between pathological gamblers (score of 5 or greater) and problem gamblers (score of 3 or 4). SOGS reliability has been tested through test-retest correlation which was $0.71(p<0.001$; Lesieur and Blume, 1987).

The Barratt Impulsiveness Scale (BIS-11) is a 30-item selfreport instrument that uses a four-point Likert scale from Rarely/Never to Almost Always (Patton et al., 1995). A total score is calculated by summing three subscale scores. The subscales are Motor ("I act on the spur of the moment"), Attentional ("I have outside thoughts when thinking"), and Non-planning ("I plan trips well ahead of time" reverse scored). The total score is reliable (in terms of internal consistency, Cronbach's $\alpha=0.86$ ).

The Boredom Proneness Scale (BPS) is a 28 -item self-report instrument that uses a five-point Likert scale from highly disagree to highly agree (Farmer and Sundberg, 1986). A total score is calculated by summing five subscales. The subscales are External Stimulation ("When I was young, I was often in monotonous and tiresome situations"), Internal Stimulation ("It is easy for me to concentrate on my activities" reverse scored), Affect ("Frequently when I am working I find myself worrying about other things"), Time Perception ("Time always seems to be passing slowly"), and Constraint ("I am good at waiting patiently" reverse scored). The total score has good reliability (Cronbach's $\alpha=0.86$ ).

The TAQ is a 42-item self-report questionnaire that assesses an individual's personal positive and negative experiences using a four-point intensity scale and across four life stages (ages 0-6, 7-12-13-18, adult; Saleptsi et al., 2004). We used a slightly modified version of the TAQ whereby we assessed only two life stages (0-12 and 13-18). Therefore, are unable to differentiate early and late childhood. In this study, we report only scores from the scales representing the Trauma factor (physical abuse, sexual abuse, witnessing, and other traumas; Saleptsi et al., 2004) from childhood (ages 0-12). We constructed a four-level variable representing levels of trauma that split the sample into similarly sized groups (i.e., none, low, mid, and high).

\section{DNA EXTRACTION AND GENOTYPING}

DNA was extracted using the DNeasy Blood and Tissue Kit (Qiagen Inc., Valencia, CA, USA) according to the manufacturer's instructions for tissue extraction with the following exceptions: the optional addition of RNase A, the elution buffer was warmed to $60^{\circ} \mathrm{C}$ before being added to the column to increase the binding affinity of the DNA to the elution buffer, two elusions were made into separate tubes using $100 \mu \mathrm{l}$ of elution buffer in each tube, and the first elution was incubated at room temperature for $10 \mathrm{~min}$ while the second was incubated for $20 \mathrm{~min}$.

The 5-HTTLPR VNTR and rs25531 SNP were amplified using primers: F: 5'-TCCTCCGCTTTGGCGCCTCTTCC-3' and R: $5^{\prime}$ TGGGGGTTGCAGGGGAGATCCTG-3'. PCR reactions $(25 \mu \mathrm{l})$ contained $5 \mu \mathrm{l}$ GoTaq Flexi buffer, $5 \mathrm{nM}$ dNTPs, $0.625 \mathrm{U}$ Taq Polymerase, $200 \mathrm{nM}$ final concentration of each primer, $0.25 \mathrm{mM}$ $\mathrm{MgCl}_{2}$, and $\sim 25 \mathrm{ng}$ of DNA. PCR conditions were followed as previously described (Wendland et al., 2006). After amplification $7 \mu \mathrm{l}$ of the PCR products were digested with 7.5 units of HpaII enzyme (New England BioLabs), $1 \times$ NE buffer 1 , and $1 \times$ BSA for $4 \mathrm{~h}$ at $37^{\circ} \mathrm{C}$. Digested products $(18 \mu \mathrm{l})$ were then run next to PCR products $(4 \mu \mathrm{l})$ on a $3.5 \%$ agarose gel at $160 \mathrm{~V}$ for $1 \mathrm{~h}$ and visualized 
under UV light with ethidium bromide stain. Fragment sizes associated with each genotype are $S_{A}=469 \mathrm{bp}, S_{G}=402$ and $67 \mathrm{bp}$, $\mathrm{L}_{\mathrm{A}}=512 \mathrm{bp}$, and $\mathrm{L}_{\mathrm{G}}=402$ and $110 \mathrm{bp}$. We randomly selected 38 samples and re-genotyped them for 5-HTTLPR and rs25531 to check for genotyping errors. As with all of our genotype calls, these were independently scored by at least two individuals, and no discrepancies were found.

\section{STATISTICAL ANALYSIS}

All analyses were conducted with IBM SPSS (version 19). We examined bivariate correlations among study variables as descriptive statistics. In our analyses, genotype groupings for 5-HTTLPR (i.e., $\mathrm{L} / \mathrm{L}$ vs. S/_) and $\mathrm{rs} 25531\left(\mathrm{~L}_{\mathrm{A}} / \mathrm{L}_{\mathrm{A}}\right.$ vs. all others) is consistent with most recent work and with current the understanding of their effects on transcription. Many of the inferential statistical tests that we conducted were not completely independent and therefore determining the exact critical alpha level to determine statistical significance is rather difficult. Because IGT performance across blocks represents repeated measurements of the same outcome across a single testing session, we used repeated-measures ANOVA to control for non-independence of scores. Additionally, 5 -HTTLPR and rs25531 genotypes are highly correlated ( $r=0.86$, $p<0.001)$ and therefore association tests with them are not independent. One may consider the 5-HTTLPR and IGT Net Score repeated-measures ANOVA that we conducted to be independent of the childhood trauma and IGT Net Score repeated-measures ANOVA and therefore it is reasonable to correct the alpha level to $0.05 / 2=0.025$ for those comparisons.

\section{RESULTS}

\section{DESCRIPTIVE STATISTICS}

Genotype frequencies for the 391 Caucasian participants are presented in Table 1. All frequencies were in Hardy-Weinberg Equilibrium and allele frequencies were consistent with prior reports. Although other genotypes were assayed for our larger study on health-risk behaviors, we restricted our analyses of IGT performance to two serotonin transporter polymorphisms.

Correlations among outcome measures and descriptive statistics are presented in Table 2 . Scores in Block 1 were not significantly correlated with other IGT performance measures with the exception of modest negative correlations with Block $2 r(389)=-0.11$, $p<0.05$, Block $4 r=-0.18, p<0.01$, and Block $5 r=-0.18$, $p<0.01$, and with Deck B $r=-0.13, p<0.01$. Performance on

Table 1 | Genotype data for the $N=391$ Caucasian participants at two serotonin transporter polymorphisms.

\begin{tabular}{lcc}
\hline Measure & 5-HTTLPR & rs25531 \\
\hline Genotype frequencies & $\mathrm{L} / \mathrm{L}=129$ & $\mathrm{~A} / \mathrm{A}=337$ \\
& $\mathrm{~L} / \mathrm{S}=183$ & $\mathrm{~A} / \mathrm{G}=51$ \\
Allele frequencies & $\mathrm{S} / \mathrm{S}=79$ & $\mathrm{G} / \mathrm{G}=3$ \\
& $\mathrm{~L}=0.56$ & $\mathrm{~A}=0.93$ \\
Hardy-Weinberg Equilibrium $\left(\chi^{2}\right)$ & $\mathrm{S}=0.44$ & $\mathrm{G}=0.07$ \\
\hline
\end{tabular}

Both markers are in Hardy-Weinberg Equilibrium ( $p>0.05)$.
IGT Blocks 2-5 were all positively correlated with each other $r \geq 0.32, p<0.01$ and with IGT Winnings $r \geq 0.64, p<0.01$ and Net Total $r \geq 0.67, p<0.01$. This pattern of correlations is strong evidence that performance on Block 1 represents a different type of decision making than that seen in other IGT blocks (i.e., ambiguity vs. risk).

To determine the trial number at which the shift from ambiguity to risk occurs (i.e., when participants appear to understand which decks are "good" and which are "bad") we set choices from decks $\mathrm{A}^{\prime}$ and $\mathrm{B}^{\prime}$ to equal -1 and choices from $\mathrm{C}^{\prime}$ and $\mathrm{D}^{\prime}$ to equal +1 for our entire sample with IGT scores $(N=473)$ and graphed the mean probability of choosing from a "good" deck across 100 trials (see Figure 1). The mean probability of choosing from a "good" deck was under 50\% until trial 19 (45\%); it then hits 56\% at trial 20 and then remained well above $50 \%$ for the remaining trials.

Iowa gambling task performance was not correlated with scores on the SOGS or on the Gambling Attitudes and Beliefs Scale $(r \leq 0.09, p \geq 0.05)$. IGT performance was also not correlated with three measures of impulsivity including SSRT, time estimation, or boredom proneness. There were modest negative correlations between scores on the BIS and IGT Winnings $r=-0.10, p<0.05$, Net Total $r=-0.13, p<0.01$, Block $1 r=-0.14, p<0.01$ and 4 $r=-0.12, p<0.05$ performance, and choices from disadvantageous decks $\mathrm{A}^{\prime} r=0.11, p<0.05$ and $\mathrm{B}^{\prime} r=0.11, p<0.05$. Therefore, certain types of impulsivity appear to play a minor role in IGT performance.

\section{IGT NET SCORE}

To replicate our previous findings (Stoltenberg and Vandever, 2010) we conducted a multiple regression with IGT Net Score [i.e., $(C+D)-(A+B)]$ in the first block (i.e., trials $1-20)$ as the dependent variable, age as a covariate and gender, 5-HTTLPR genotype (L/L vs. S/_) and their interaction as independent variables. The was no main effect for gender $F(1,386)=0.41, p=0.52$, but there was a significant effect of 5-HTTLPR genotype $F(1$, $386)=8.18, p=0.004$, partial $\eta^{2}=0.021$, and no significant gender $\times$ genotype interaction $F(1,386)=0.46, p=0.50$. Although we did not replicate the gender moderation of the 5-HTTLPR effect on decision making under ambiguity, we found strong evidence that, on average, individuals with the L/L genotype choose more cards from the "bad" decks in the first 20 trials of the IGT (see Figure 2).

To be consistent with our previous analyses and to examine these effects across the five IGT blocks, we then conducted repeated-measures ANOVA with IGT Net scores across five blocks as the dependent variables and with gender, 5-HTTLPR genotype and their interaction as factors. We also included age as a covariate. Overall IGT Net scores (i.e., pooled across blocks) were examined with between subject analysis, which indicated no significant main effects for gender, $F(1,384)=1.88, p=0.17$, or genotype $F(1,386)=0.32, p=0.57$, and no significant gender $\times$ genotype interaction $F(1,386)=0.005, p=0.95$.

To examine the patterns of change across IGT blocks we examined within-subject analyses. There was a significant effect of Block $F(3.19,1230.86)=5.47, p=0.001$, and a significant Block $\times$ Genotype interaction $F(3.19,1230.86)=3.67$, 


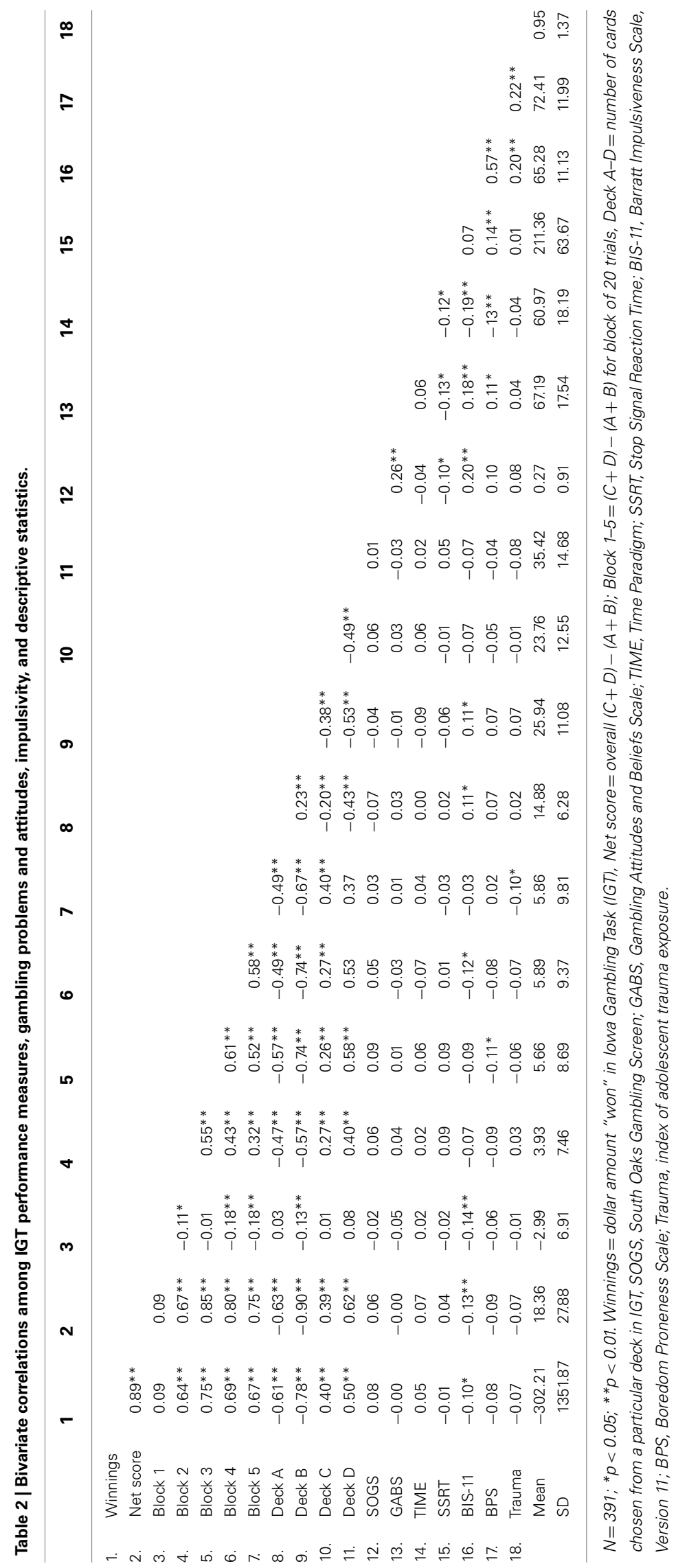




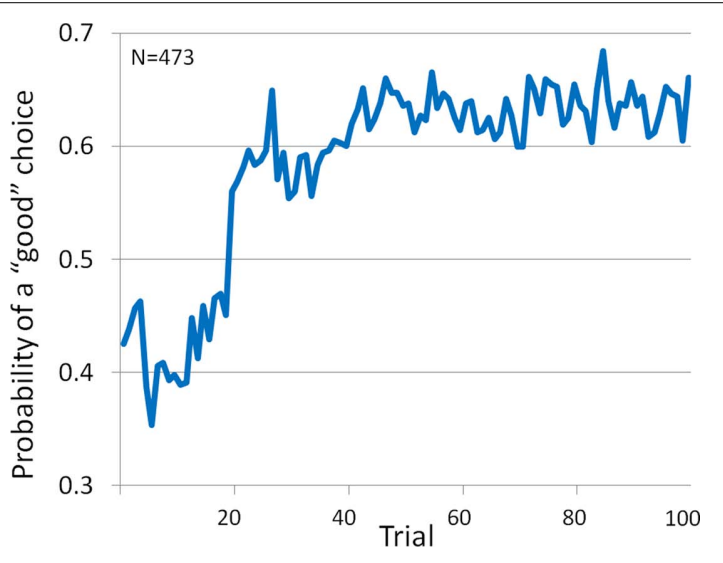

FIGURE 1 | Probability of choosing a "good" card (i.e., from decks C' and $D^{\prime}$ ) across 100 trials.

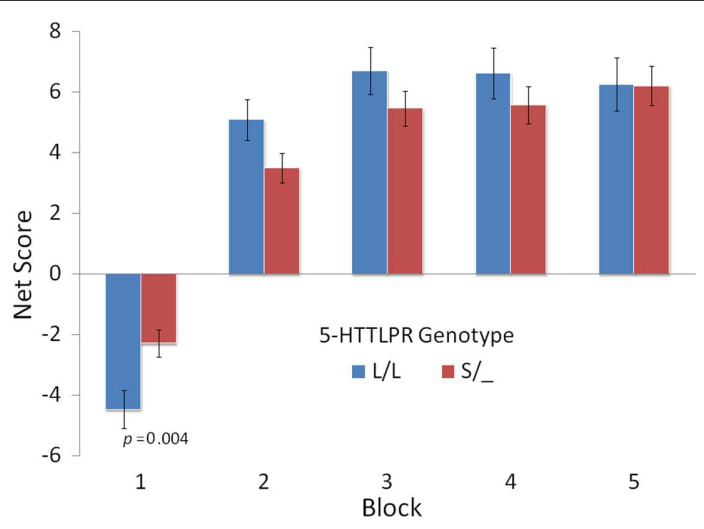

FIGURE 2 | Mean net score $\left[\left(C^{\prime}+D^{\prime}\right)-\left(A^{\prime}+B^{\prime}\right)\right]$ for groups defined by 5-HTTLPR genotype across five blocks of 20 trials.

$p=0.01$ (for all repeated-measures results, we do not assume Sphericity and report the Huynh-Feldt corrected $\mathrm{d} f$ and $p$ values). Neither the Block $\times$ Gender $F(3.19,1230.86)=1.75, p=0.15$, nor the Block $\times$ Gender $\times$ Genotype interaction effect $F(3.19$, 1230.86) $=0.95, p=0.42$, were significant.

When we carried out these comparisons with net scores pooled across Blocks 1 and 2 (i.e., the first 40 trials) and Blocks 3-5 (i.e., the last 40 trials) for comparability with He et al. (2010), there were no significant main effects or interactions although the pattern of mean scores resembled that shown in (He et al., 2010). This result suggests that pooling the first two blocks obscures the elevated number of risky choices made by $\mathrm{L} / \mathrm{L}$ individuals and given our evidence that Blocks 1 and 2 are negatively correlated and that the probability of choosing a good deck is negative for only the first 19 trials we recommend that future studies analyze Block 1 separately from the others to distinguish ambiguity vs. risk.

Identical repeated-measure ANOVAs were carried out for IGT net scores to examine the effects of the 5-HTTLPR triallelic genotype (5-HTTLPR and rs25531). We defined the triallelic genotype groups as $0=\mathrm{LA} / \mathrm{LA}, 1=\mathrm{LA} / \mathrm{LG}, \mathrm{LA} / \mathrm{S}, \mathrm{LG} / \mathrm{LG}, \mathrm{S} \_/ \mathrm{S} \_$. With the exception of the expected effect of Block in within-subject

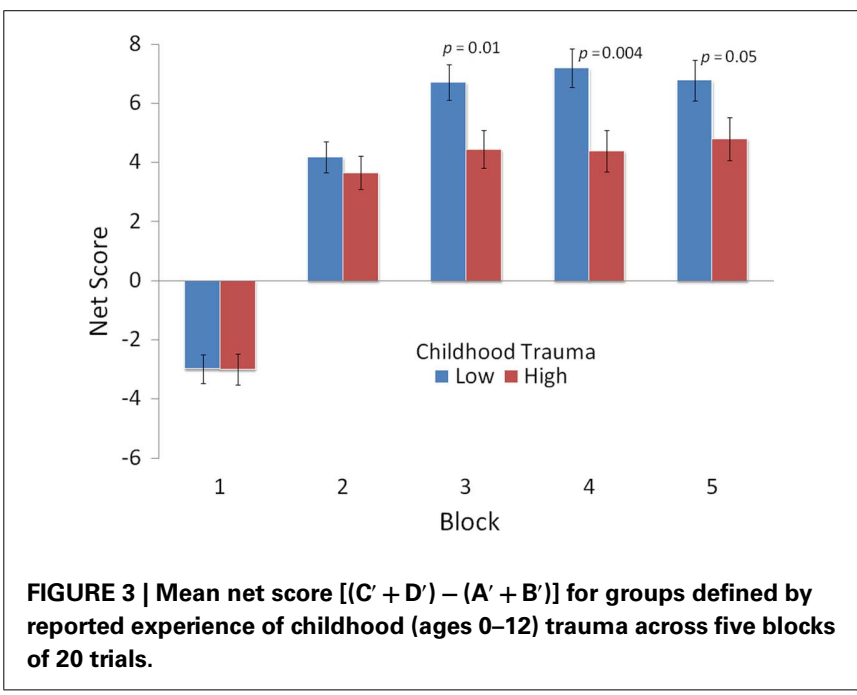

analyses, no other main effects or interactions were significant. Block $\times$ rs25531 genotype $F(3.18,77.73)=1.19, p=0.31$, partial $\eta^{2}=0.003$. It appears that considering the rs 25531 polymorphism in 5-HTTLPR weakens the association between genotype and IGT performance. Therefore, we focus the remaining analyses on 5-HTTLPR.

To assess the effects of childhood trauma on IGT performance and to examine potential 5-HTTLPR genotype by childhood trauma interactions we conducted repeated-measures ANOVA as before, but with the addition of the four-level variable of childhood trauma as a factor. Tests of within-subject effects again indicated the significant main effect of Block $F(2.66$, $1235.01)=2.66, p=0.04$, and a Block $\times$ Genotype interaction $F(3.30,1235.01)=2.63, p=0.04$. In addition, there was a significant interaction effect of Block $\times$ Childhood Trauma $F(9.91$, $1235.01)=2.05, p=0.03$. No other effects in the model were significant. Post hoc between subjects tests indicated that means of groups defined by a median split of the childhood trauma variable had significant different IGT net scores in Blocks 3-5 (see Figure 3). The only test of between subjects effects to reach significance was Childhood Trauma $F(3,374)=3.22, p=0.02$. Different patterns of IGT performance across blocks were associated with level of Childhood Trauma, as was the overall IGT performance. This repeated-measures analysis indicated that childhood Trauma did not significantly interact with 5-HTTLPR genotype or gender in its effects on IGT performance.

\section{DISCUSSION}

We found genetic variation at the 5-HTTLPR polymorphism to be associated with decision making under ambiguity (trials 1-20), but not under risk (trials 21-100). Further, we observed a strong association between the self-reported experience of childhood trauma and decision making under risk, but not under ambiguity. Our findings add to the growing literature on genetically based individual differences in decision making and on the effects of early life stress on later life outcomes.

Consistent with other studies of decision making in samples of healthy, non-patient adults, we found that individuals learned 
to select cards from the "good" decks as the task progressed. We also present strong evidence that only the first 20 trials should be considered decision making under ambiguity (i.e., insufficient information regarding task contingencies), at least for healthy adults. Although it may be argued that trials 21-40 do not clearly represent decision making under risk (i.e., when task contingencies are explicitly known), they also do not represent decision making under ambiguity as seen in trials 1-20 (i.e., Block 1). It might be reasonable to describe trials $21-40$ as a transition phase of decision making that represents a state of knowing prior to certainty. In our relatively large sample, the mean probability of choosing a card from the "good" decks was below 50\% for trials 1-19 and above $50 \%$ for all of the remaining 80 trials (i.e., trial $19=45 \%$ and trial $20=56 \%$ ). Recent studies are consistent with this characterization as trials 1-20 representing ambiguity and trials 41-100 representing risk (Brand et al., 2007b;Gendle and Golding, 2010; Stoltenberg and Vandever, 2010).

We undertook the present study to replicate and extend our earlier work (Stoltenberg and Vandever, 2010) with an independently collected and larger sample size. Our study sample is the largest group of Caucasians tested in the literature. We replicated an association of 5-HTTLPR genotype and decision making under risk, but did not observe evidence to support the gender moderation of the 5-HTTLPR association that we observed previously. The most likely interpretation of this failure to replicate the gender by genotype interaction effect is that the smaller sample size in our previous study resulted in unreliable estimates of the mean scores of women. We observed the same pattern of results for men, so performance variability in women is the simplest explanation. It may be that a small number of outliers in our first study biased our estimates of the mean net score. In neither study did we assess nor control for menstrual cycle status.

Homozygosity for the L allele of 5-HTTLPR was associated with choosing more cards from the "bad" decks in the first 20 trials (i.e., Block 1). In other words, individuals with the L/L genotype were initially more risk taking than $S$ allele carriers. These findings are consistent with the position that although the $S$ allele is generally considered to be the risky allele for psychopathology, it may predispose carriers to behave in less risky ways than $\mathrm{L} / \mathrm{L}$ homozygotes and can be considered to be adaptive in certain contexts (Belsky et al., 2009; Homberg and Lesch, 2011). When the 5-HTTLPR triallelic genotype was examined (i.e., 5-HTTLPR and rs25531) genotype was not associated individual differences in IGT performance. We found that performance on Block 1 was not correlated with gambling problems or problematic attitudes and beliefs about gambling. Block 1 performance was also not correlated with response inhibition, time estimation, boredom proneness, or childhood trauma. In addition, a recent study reported that performance on Block 1 is not associated executive functions such as set shifting, categorization, or cognitive flexibility (Brand et al., 2007a). Our finding that 5-HTTLPR genotype is associated with risk taking under ambiguity appears to be consistent with a recent study that reported manipulation of 5-HT function by ingestion of the serotonin precursor 5-HTP affected risk taking in Block 1 of the IGT, but not in subsequent blocks (Gendle and Golding, 2010). Tryptophan depletion, which reduces serotonin availability, also affected risk taking with a greater impact on those with the L/L genotype (Blair et al., 2008). The pattern of influence that 5-HT availability has on risk taking appears to be complex and is likely dependent on the task and may be moderated by gender, country of origin (i.e., genetic and/or cultural background). Both raising and lowering 5-HT availability appears to increase risk taking as does the 5-HTTLPR genotype associated with greater transcriptional activity of the SLC6A4 gene, greater numbers of transporters and higher 5-HT reuptake. It seems safe to say that 5-HT function affects decision making, but simple explanations are likely to be inadequate. It is likely that to fully characterize the relations between 5 -HT function and decision making phenotypes that are more decomposable into specific constructs will need to be studied (Schonberg et al., 2011).

We found that the experience of relatively higher levels of childhood trauma is associated worse performance on the last 60 trials of the IGT. We would like to note that the $p$-value for the between subjects tests was 0.02 and we would like to consider this nominal evidence for association and suggest that this finding should be replicated. Performance on the last 60 trials can be considered to represent decision making under risk. That is, participants are likely to know the response contingencies during those trials. These findings are consistent with studies that have identified deficits in cognitive control and changes in brain structure and function as a result of early life stress (Ivy et al., 2010; Mueller et al., 2010). The effect of childhood trauma may be mediated by binge drinking, which has been shown to affect performance on the IGT (Goudriaan et al., 2007), or by other mechanisms. Subsequent studies should examine the potential pathways through which the experience of childhood trauma might influence decision making under risk.

This study has several strengths. The size of our study sample is rather large and is relatively homogeneous with respect to age, race, and ethnicity. Our approach was strongly driven by hypotheses and we did not examine other potential genetic associations. Our findings replicate the major finding of our previous study in an independent sample (Stoltenberg and Vandever, 2010). These two studies are the only ones we are aware of to examine these associations in US Caucasians. We appear to be the first to report the negative effect of the experience of childhood trauma on decision making under risk as indexed by the IGT (trials 41-100). Subsequent efforts should be made to replicate this finding and to better characterize the factors influencing the effect with regards to timing and type of childhood trauma. We also show that impulsivity (variously defined) and gambling problems are not associated with differences on the IGT. We examined the rs25531 polymorphism in addition to 5-HTTLPR, but did not find it to be associated with IGT performance.

This study also has some weaknesses. We did not screen participants for mental illness, brain trauma, or menstrual cycle. We did not examine other genetic polymorphisms that may contribute to individual differences in decision making. Significance levels of findings reported here should be considered in the context of the direct replication that we undertook and that we did make several statistical comparisons overall. We did not conduct additional tests of cognitive ability (e.g., short term memory) or decision 
making that would enable us to better characterize the observed associations. We are in the process of conducting a follow-up study in which we are collecting short term memory and risk aversion assessments.

\section{CONCLUSION}

Our study replicates our previous finding that homozygosity for the $\mathrm{L}$ allele of 5-HTTLPR is associated with increased risk taking under ambiguity. We also showed that the experience of childhood trauma adversely affected decision making under risk. Our findings indicate that only the first 20 trials of the IGT should be

\section{REFERENCES}

Anda, R. F., Felitti, V. J., Bremner, J. D., Walker, J. D., Whitfield, C., Perry, B. D., Dube, S. R., and Giles, W. H. (2006). The enduring effects of abuse and related adverse experiences in childhood. A convergence of evidence from neurobiology and epidemiology. Eur. Arch. Psychiatry Clin. Neurosci. 256, 174-186.

Bechara, A. (2007). Iowa Gambling Task Professional Manual. Lutz, FL: Psychological Assessment Resources, Inc.

Bechara, A., Damasio, H., Tranel, D., and Damasio, A. R. (1997). Deciding advantageously before knowing the advantageous strategy. Science 275, 1293-1295.

Bechara, A., Tranel, D., and Damasio, H. (2000). Characterization of the decision-making deficit of patients with ventromedial prefrontal cortex lesions. Brain $123(\mathrm{Pt}$ 11), 2189-2202.

Belsky, J., Jonassaint, C., Pluess, M., Stanton, M., Brummett, B., and Williams, R. (2009). Vulnerability genes or plasticity genes? Mol. Psychiatry 14, 746-754.

Blair, K. S., Finger, E., Marsh, A. A., Morton, J., Mondillo, K., Buzas, B., Goldman, D., Drevets, W. C., and Blair, R. J. (2008). The role of 5-HTTLPR in choosing the lesser of two evils, the better of two goods: examining the impact of 5-HTTLPR genotype and tryptophan depletion in object choice. Psychopharmacology (Berl.) 196, 29-38.

Brand, M., Grabenhorst, F., Starcke, K., Vandekerckhove, M. M., and Markowitsch, H. J. (2007a). Role of the amygdala in decisions under ambiguity and decisions under risk: evidence from patients with UrbachWiethe disease. Neuropsychologia 45, 1305-1317.

Brand, M., Recknor, E. C., Grabenhorst, F., and Bechara, A. (2007b). Decisions under ambiguity and decisions under risk: correlations with executive functions and comparisons of two different gambling tasks with implicit and explicit rules. $J$. Clin. Exp. Neuropsychol. 29, 86-99.

Brand, M., Labudda, K., and Markowitsch, H. J. (2006). Neuropsychological correlates of decision-making in ambiguous and risky situations. Neural Netw. 19, 1266-1276.

Caspi, A., Hariri, A. R., Holmes, A., Uher, R., and Moffitt, T. E. (2010). Genetic sensitivity to the environment: the case of the serotonin transporter gene and its implications for studying complex diseases and traits. Am. J. Psychiatry 167, 509-527.

Caspi, A., Sugden, K., Moffitt, T. E., Taylor, A., Craig, I. W., Harrington, H., Mcclay, J., Mill, J., Martin, J., Braithwaite, A., and Poulton, R. (2003). Influence of life stress on depression: moderation by a polymorphism in the 5-HTT gene. Science 301, 386-389.

Da Rocha, F. F., Malloy-Diniz, L., Lage, N. V., Romano-Silva, M. A., De Marco, L. A., and Correa, H. (2008). Decision-making impairment is related to serotonin transporter promoter polymorphism in a sample of patients with obsessivecompulsive disorder. Behav. Brain Res. 195, 159-163.

Dougherty, D. M., Mathias, C. W., Marsh, D. M., and Jagar, A. A. (2005). Laboratory behavioral measures of impulsivity. Behav. Res. Methods 37, 82-90.

Farmer, R., and Sundberg, N. D. (1986). Boredom proneness - the development and correlates of a new scale. $J$. Pers. Assess. 50, 4-17.

Gendle, M. H., and Golding, A. C. (2010). Oral administration of 5-hydroxytryptophan (5-HTP) impairs decision making under ambiguity but not under risk: evidence from the Iowa Gambling Task. Hum. Psychopharmacol. 25, 491-499.

Glimcher, P. W., Camerer, C., Poldrack, R. A., and Fehr, E. (eds). (2008).

considered the ambiguity phase of the task. Subsequent efforts to delineate the factors underlying decision making should focus on tightening the phenotype and should assess cognitive measures as well as early life influences.

\section{ACKNOWLEDGMENTS}

This study was made possible by R15MH077654-01A1 (NIMH) and by P20 RR016479 from the National Center for Research Resources (NCRR), a component of the National Institutes of Health (NIH).

Neuroeconomics: Decision Making and the Brain. London: Academic Press.

Goudriaan,A. E., Grekin, E. R., and Sher, K. J. (2007). Decision making and binge drinking: a longitudinal study. Alcohol. Clin. Exp. Res. 31, 928-938.

Ha, R. Y., Namkoong, K., Kang, J. I., Kim, Y. T., and Kim, S. J. (2009). Interaction between serotonin transporter promoter and dopamine receptor D4 polymorphisms on decision making. Prog. Neuropsychopharmacol. Biol. Psychiatry 33, 1217-1222.

He, Q., Xue, G., Chen, C., Lu, Z. Dong, Q., Lei, X., Ding, N., Li, J., Li, H., Moyzis, R. K., and Bechara, A. (2010). Serotonin transporter gene-linked polymorphic region (5HTTLPR) influences decision making under ambiguity and risk in a large Chinese sample. Neuropharmacology 59, 518-526.

Heim, C., and Nemeroff, C. B. (2002). Neurobiology of early life stress: clinical studies. Semin. Clin. Neuropsychiatry 7, 147-159.

Hillis, S. D., Anda, R. F., Felitti, V. J., and Marchbanks, P. A. (2001). Adverse childhood experiences and sexual risk behaviors in women: a retrospective cohort study. Fam. Plann. Perspect. 33, 206-211.

Homberg, J. R., and Lesch, K. P. (2011). Looking on the bright side of serotonin transporter gene variation. Biol. Psychiatry 69, 513-519.

Homberg, J. R., Van Den Bos, R. Den Heijer, E., Suer, R., and Cuppen, E. (2008). Serotonin transporter dosage modulates long-term decision-making in rat and human. Neuropharmacology 55, 80-84.

Ivy, A. S., Rex, C. S., Chen, Y., Dube, C., Maras, P. M., Grigoriadis, D. E., Gall, C. M., Lynch, G., and Baram, T. Z. (2010). Hippocampal dysfunction and cognitive impairments provoked by chronic earlylife stress involve excessive activation of CRH receptors. J. Neurosci. 30, 13005-13015.
Juhasz, G., Downey, D., Hinvest, N., Thomas, E., Chase, D., Toth, Z. G., Lloyd-Williams, K., Mekli, K., Platt, H., Payton, A., Bagdy, G., Elliott, R., Deakin, J. F., and Anderson, I. M. (2010). Risk-taking behavior in a gambling task associated with variations in the tryptophan hydroxylase 2 gene: relevance to psychiatric disorders. Neuropsychopharmacology 35, 1109-1119.

Lesieur, H. R., and Blume, S. B. (1987). The South Oaks Gambling Screen (SOGS): a new instrument for the identification of pathological gamblers. Am. J. Psychiatry 144, 1184-1188.

Logan, G. D., Schachar, R. J., and Tannock, R. (1997). Impulsivity and inhibitory control. Psychol. Sci. 8 , 60-64.

Maurex, L., Zaboli, G., Wiens, S., Asberg, M., Leopardi, R., and Ohman, A. (2009). Emotionally controlled decision-making and a gene variant related to serotonin synthesis in women with borderline personality disorder. Scand. J. Psychol. 50, 5-10.

Mueller, S. C., Maheu, F. S., Dozier, M., Peloso, E., Mandell, D., Leibenluft, E., Pine, D. S., and Ernst, M. (2010). Early-life stress is associated with impairment in cognitive control in adolescence: an fMRI study. Neuropsychologia 48, 3037-3044.

Must, A., Juhasz, A., Rimanoczy, A., Szabo, Z., Keri, S., and Janka, Z. (2007). Major depressive disorder, serotonin transporter, and personality traits: why patients use suboptimal decision-making strategies? J. Affect. Disord. 103, 273-276.

Overman, W. H., Boettcher, L., Watterson, L., and Walsh, K. (2010). Effects of dilemmas and aromas on performance of the Iowa gambling task. Behav. Brain Res. 218, 64-72.

Patton, J. H., Stanford, M. S., and Barratt, E. S. (1995). Factor structure of the Barratt impulsiveness scale. J. Clin. Psychol. 51, 768-774. 
Rogers, R. D. (2011). The roles of dopamine and serotonin in decision making: evidence from pharmacological experiments in humans. Neuropsychopharmacology 36, 114-132.

Saleptsi, E., Bichescu, D., Rockstroh, B., Neuner, F., Schauer, M., Studer, K., Hoffmann, K., and Elbert, T. (2004). Negative and positive childhood experiences across developmental periods in psychiatric patients with different diagnoses - an explorative study. BMC Psychiatry 4, 40. doi: 10.1186/1471-244X-4-40

Schonberg, T., Fox, C. R., and Poldrack, R. A. (2011). Mind the gap: bridging economic and naturalistic risk-taking with cognitive neuroscience. Trends Cogn. Sci. (Regul. Ed.) 15, 11-19.

Stoltenberg, S. F., and Vandever, J. M. (2010). Gender moderates the association between 5-HTTLPR and decision-making under ambiguity but not under risk. Neuropharmacology 58, 423-428.

Strong, D. R., Daughters, S. B., Lejuez, C. W., and Breen, R. B. (2004). Using the Rasch model to develop a revised gambling attitudes and beliefs scale (GABS) for use with male college student gamblers. Subst. Use Misuse 39, 1013-1024.

Wendland, J. R., Martin, B. J., Kruse, M. R., Lesch, K. P., and Murphy,
D. L. (2006). Simultaneous genotyping of four functional loci of human SLC6A4, with a reappraisal of 5-HTTLPR and rs25531. Mol. Psychiatry 11, 224-226.

Conflict of Interest Statement: The authors declare that the research was conducted in the absence of any commercial or financial relationships that could be construed as a potential conflict of interest.

Received: 31 March 2011; accepted: 01 June 2011; published online: 13 June 2011.

Citation: Stoltenberg SF, Lehmann $M K$, Anderson $C$, Nag $P$ and
Anagnopoulos C (2011) Serotonin transporter (5-HTTLPR) genotype and childhood trauma are associated with individual differences in decision making. Front. Gene. 2:33. doi: 10.3389/fgene.2011.00033

This article was submitted to Frontiers in Behavioral and Psychiatric Genetics, a specialty of Frontiers in Genetics.

Copyright $\odot 2011$ Stoltenberg, Lehmann, Anderson, Nag and Anagnopoulos. This is an open-access article subject to a nonexclusive license between the authors and Frontiers Media SA, which permits use, distribution and reproduction in other forums, provided the original authors and source are credited and other Frontiers conditions are complied with. 